\title{
TAIL EQUIVALENT LINEARIZATION METHODS FOR SEISMIC RESPONSE SPECTRUM ANALYSIS
}

\author{
U. Alibrandi ${ }^{1,2}$ \\ ${ }^{1}$ Nanyang Tecnological University, Department of Electrical and Electronic Engineering \\ ${ }^{2}$ Sinberbest - SINgapore-BERkeley Building Efficiency and Sustainability in the Tropics \\ 1 Create Way, Create Tower, 138062 Singapore \\ e-mail: umbertoalibrandi@ntu.edu.sg
}

Keywords: Performance Based Earthquake Engineering, Stochastic Dynamic Analysis, Eartquake risk assessment, Response Spectrum compatible Power Spectral Density, FORM, Tail Equivalent Linearization Method

\begin{abstract}
This paper aims to conduct seismic reliability analysis of systems using stochastic ground motion model coherent with the codes. This is achieved by developing a Power Spectral Density (PSD) which is compatible with the response spectrum, for assigned valued of seismic zone, soil category and limit state. The seismic reliability analysis is developed inside the most general framework of Performance Based Earthquake Engineering (PBEE) and adopts the tools of the Stochastic Dynamic Analysis. It has been adopted the FORM approximation because of its computational simplicity and effectiveness. FORM allows also to define the Tail Equivalent Linearization System (TELS), which is an equivalent linear system having for each threshold the same design point of the original nonlinear system. The application of the linear theory of the random vibrations to the TELS allows to determine the first-passage probability of the seismic demand, without requiring no further dynamic computations with respect to FORM.
\end{abstract}




\section{INTRODUCTION}

The response spectrum is the most used tool by practical engineers for a basic representations of the seismic action. The ordinary structures are designed to behave inelastically for the seismic intensity prescribed by the design codes. This is obtained in the framework of the response-spectrum based analysis through the adoption of the behavior factor. However, a realistic evaluation of the structural response should be dynamic and related to the damage that occurs under repeated and usually inelastic cycling. This because the philosophy of earthquake-resistant design is not to prevent the occurrence of the damage, but to limit its occurrence under the design earthquake [1]. Starting from these considerations, damage-based limit states along with sophisticated inelastic structural models are needed.

Moreover it is largely recognized that the natural hazards and the strengths of the materials are subjected to several sources of uncertainty. Typically the existing codes take into account the uncertainties through the introduction of suitable partial safety factors. It is however well known that they are valid only for the most general case and moreover they are usually overly conservative. Therefore, a probabilistic analysis is essential in predicting the most likely behavior of the building. These tasks can be accomplished by using the general probabilistic framework of Performance Based Earthquake Engineering (PBEE), originally proposed by Cornell and Krawinkler [2] whose main steps are: (i) characterization and assessment of the seismic hazard, (ii) probabilistic assessment of the seismic demand on the structure, (iii) probabilistic assessment of the resulting physical damage, (iv) assessment of expected economic and other losses resulting from damage [3-5]. The present paper only addresses steps (i) and (ii) of this framework.

In the current PBEE practice, the seismic hazard is characterized in terms of one or more intensity measures ( $I M)$, for which annual probabilities of exceedance are developed. For each IM level corresponding to a specified annual rate of exceedance, the seismic demand is determined through the conditional probability distribution of the Engineering Demand Parameter ( $E D P$ ) on the structure (e.g. interstory drift). This is usually done by nonlinear timehistory analyses with a selected suite of recorded ground motions which are individually scaled to the specified IM level [6]. The computed sample of the maximum responses is then used to estimate the median and logarithmic standard deviation of the seismic demand, to which a lognormal distribution is fitted. Denoting $X_{\max }$ the maximum response of the EDP for the given intensity level $I M \equiv i m$, the result of the analysis is the Probability Of Exceedance (POE) $P[x \mid i m]=\operatorname{Prob}\left[X_{\max }>x \mid I M=i m\right]$, also known in literature as fragility curve.

In the existing codes the seismic hazard is defined by suitable response spectra, whose parameters take into account of the site conditions, while the intensity measure $I M$ is given by the Peak Ground Acceleration $A_{g}$, defined for each site and for different limit states. When the behavior of the system is highly nonlinear, the technique of the response spectrum cannot be applied. In these cases the codes allow to model the seismic action through groundacceleration time-history analyses, to which is required to match the elastic response spectra for a viscous damping $\zeta_{0}=5 \%$.

In this paper, following Der Kiureghian and Fujimura [7] the seismic reliability analysis is developed following a fully stochastic approach: (i) the ground motion is defined as a stochastic process instead of a suite of scaled, recorded ground motions, (ii) the conditional POE $P\left[x \mid a_{g}\right]=\operatorname{Prob}\left[X_{\max }>x \mid I M=a_{g}\right]$ is determined through the tools of stochastic dynamic analysis, instead of a set of nonlinear time-history analyses. This approach has several advantages. First, the codes typically require the adoption of a reduced number of sets of accel- 
erograms, which generally do not allow to develop a significant statistical analysis. Second, the codes require that the accelerograms are scaled to match the elastic response spectrum, which remains a controversial step. Conversely, in this paper the stochastic input is defined as a Power Spectral Density ( $P S D$ ) compatible with the response spectrum [8, 9]. In this way, at least for an SDOF linear system, results in terms of maximum response peak by stochastic analysis and using the Response Spectrum are almost identical. It is underlined that the PSD is related only to the ground acceleration and not to the superimposed structure, so that it is independent of the damping ratio either by yielding or by inherent nonlinearities of the superimposed structure [9].

It is here noted that the application of Stochastic Dynamic Analysis to evaluate the conditional POE $P\left[x \mid a_{g}\right]$ is equivalent to develop a statistical analysis where infinite accelerograms are taken into account. On the other hand, the application of stochastic dynamic to a general nonlinear MDOF system is very challenging. Some methods of simulations have been developed to reduce the computational effort [10], but in any case they require several thousands of analyses for each threshold. Here the stochastic analysis is developed by using the recently presented Tail Equivalent Linearization Method (TELM) [11], which is based on the well-known First-Order Reliability Method (FORM) applied to the random vibration problems [12]. Once the PSD compatible with the response spectrum has been evaluated, the stochastic ground motion is discretized into a set of normal standard random variables [13, 14], collected in the vector $\boldsymbol{u}$. Define the tail probability as the probability that stochastic response at time instant $t$ is greater than a chosen threshold $x$, that is $P_{f}=\operatorname{Prob}[X(t, u) \geq x]$. The application of FORM to the corresponding reliability problem gives a first-order approximation of the tail probability, which is accurate enough for most problems of stochastic dynamic analysis. FORM is very effective, since it requires only a low number of analysis; the main computational effort is to be attributed to the evaluation of the response gradients, which can be determined by using the Direct Differentiation Method [15, 16] implemented in some softwares like OpenSees [17]. Alternatively, some suitable free-derivative algorithms have been developed to determine the design point with reduced computational cost $[14,18]$.

An interesting property of FORM is that it gives the Tail Equivalent Linear System (TELS), defined as the equivalent linear system having for each threshold $x$ the same design point of the original nonlinear system. In this way, by applying the theory of the random vibrations to the TELS it is possible to determine, without no further computation, the first passage probability, coinciding with the fragility curve corresponding to the chosen limit state, i.e. $\operatorname{Prob}\left[X_{\max }(t, \boldsymbol{u}) \geq x\right] \equiv P\left[x \mid a_{g}\right]$.

\section{SEISMIC HAZARD ANALYSIS}

In the existing codes the seismic hazard is defined by suitable response spectra, and the intensity measure $I M$ of the seismic event is given by the Peak Ground Acceleration (PGA) $A_{g}$, defined for each site and for different limit states, e.g. Damage Limit State ( $D L S$ ) and Ultimate Limit State ( $U L S$ ). In this case the value $I M \equiv A_{g}$ together with its probability of occurrence $p(\mathrm{im})$ are defined by the codes. Let $a_{g, D L S}$ and $a_{g, U L S}$ be the suggested values by the codes of $A_{g}$ for DLS and ULS, respectively; in the Eurocodes one has $p\left(a_{g, D L S}\right)=63 \%$ and $p\left(a_{g, U L S}\right)=10 \%$, while the corresponding return periods are $T_{R, D L S}=50$ and $T_{R, U L S}=475$ years. 
In this paper the elastic acceleration response spectrum prescribed by Eurocode 8 is considered:

$$
R S A\left(T, \zeta_{0}\right)= \begin{cases}a_{g} S\left[1+\frac{T}{T_{B}}(2.5 \eta-1)\right] & 0 \leq T \leq T_{B} \\ 2.5 a_{g} S \eta & T_{B} \leq T \leq T_{C} \\ 2.5 a_{g} S \eta\left(\frac{T_{C}}{T}\right) & T_{C} \leq T \leq T_{D} \\ 2.5 a_{g} S \eta\left(\frac{T_{C} T_{D}}{T}\right) & T_{D} \leq T \leq T_{C}\end{cases}
$$

where $\operatorname{RSA}\left(T, \zeta_{0}\right)$ is the elastic response spectrum, $\eta=\left[10 /\left(5+\zeta_{0}\right)\right]^{1 / 2}, S$ is the soil factor, $T_{B}$ and $T_{C}$ are respectively the lower and upper limits of the period of the constant spectral acceleration branch, $T_{D}$ is the value defining the beginning of the constant displacement response range of the spectrum. The seismic codes allow time-history representations of the seismic action for analyzing the non-linear behavior of the structures whereas the response spectrum technique might not provide accurate results. The codes do not suggest a method for generating the earthquake time-histories, but only recommend that they are response-spectrum compatible. Many common approaches model the earthquake as realizations of a stationary Gaussian stochastic process. This kind of representation is adopted in this paper, and using the model proposed in $[8,19]$ one obtains the following handy recursive expression to determine the one-sided Power Spectral Density (PSD) compatible with the response spectrum

$$
\begin{cases}G\left(\omega_{i}\right)=0 & 0 \leq \omega \leq \omega_{\alpha} \\ G\left(\omega_{i}\right)=\frac{4 \zeta_{0}}{\pi \omega_{i}-4 \zeta_{0} \omega_{i-1}}\left(\frac{R S A\left(\omega_{i}, \zeta_{0}\right)^{2}}{\bar{\eta}_{U}^{2}\left(\omega_{i}, \zeta_{0}\right)}-\Delta \omega \sum_{k=1}^{i-1} G\left(\omega_{k}\right)\right) & \omega>\omega_{\alpha}\end{cases}
$$

where $R S A\left(\omega_{i}, \zeta_{0}\right)$ is the pseudo-acceleration response spectrum obtained from Eq.(1) for given damping ratio $\zeta_{0}$ and circular frequency $\omega_{i}=(2 \pi) / T_{i}$, and $\bar{\eta}_{U}\left(\omega_{i}, \zeta_{0}\right)$ is the peak factor under the assumption of a barrier out-crossing in clumps

$$
\bar{\eta}_{U}\left(\omega_{i}, \zeta_{0}\right)=\sqrt{2 \ln \left\{2 N_{U}\left[1-\exp \left[-\delta_{U}^{1.2} \sqrt{\pi \ln \left(2 N_{U}\right)}\right]\right]\right\}}
$$

being

$$
\left\{\begin{array}{l}
N_{U}=\frac{t}{2 \pi} \omega_{i}(-\ln \{0.5\})^{-1} \\
\delta_{U}=\left[1-\frac{1}{1-\zeta_{0}^{2}}\left(1-\frac{2}{\pi} \arctan \frac{\zeta_{0}}{\sqrt{1-\zeta_{0}^{2}}}\right)^{2}\right]^{1 / 2}
\end{array}\right.
$$

In Eq.(4) it has been assumed that the input PSD is smooth and $\zeta_{0} \ll 1$. Moreover $t$ is the time observing window, assumed equal to the time instant when the system achieves the stationarity, while $\omega_{\alpha} \cong 1 \mathrm{rad} / \mathrm{sec}$ is the lowest bound of the existence domain of $\bar{\eta}_{U}$. Once the 
response-spectrum compatible PSD $G(\omega)$ of the ground motion is evaluated through Eq.(1)(4), the stochastic ground motion model $\ddot{X}_{g}(t, u)$ can defined through the discrete Fourier series as $[13,14,20,21]$

$$
\begin{aligned}
\ddot{X}_{g}(t, \boldsymbol{u}) & =\sum_{k=1}^{n} \sqrt{G\left(\omega_{k}\right) \Delta \omega}\left[\cos \left(\omega_{k} t\right) u_{k}^{c}+\sin \left(\omega_{k} t\right) u_{k}^{s}\right]= \\
& =\sum_{k=1}^{n} s_{k}^{c}(t) u_{k}^{c}+s_{k}^{s}(t) u_{k}^{s}=s(t) \cdot \boldsymbol{u}
\end{aligned}
$$

where $n$ is the number of harmonic components, $\sigma_{k}=\sqrt{G\left(\omega_{k}\right) \Delta \omega}, k=1,2, \ldots, n$, $\Delta \omega \leq(2 \pi) / t, \quad \boldsymbol{u}=\left\{\begin{array}{ll}\boldsymbol{u}^{c} & \boldsymbol{u}^{s}\end{array}\right\}^{T}$ is an $(2 n)$ - vector of normal standard random variables,

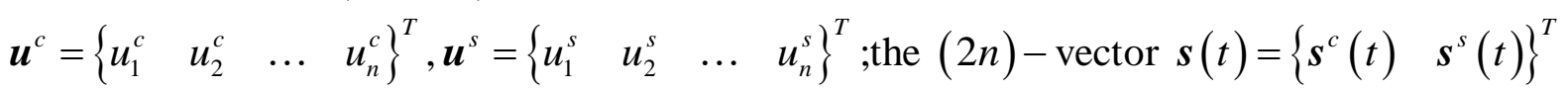
collects the corresponding shape function $s_{k}^{c}(t)=\sigma_{k} \cos \left(\omega_{k} t\right)$, and $s_{k}^{s}(t)=\sigma_{k} \sin \left(\omega_{k} t\right)$, which are determined by the correlation structure of the input given by the underlying PSD. Note that the model given by Eq.(5) is flexible because it allows us to reduce the number of random variables. If the cutoff frequency $\omega_{c}$ of the system is known, we will consider only the harmonic components $\omega_{k} \leq \omega_{c}$. Eq.(5) can be used for generating samples of the stochastic ground motion model: each realization is simply obtained through the generations of the normal standard random variables $\boldsymbol{u}$. In this paper Eq.(5) it is used for determining directly the first passage probability of the EDP using the tools of the stochastic dynamic analysis, as detailed in the next section.

\section{STRUCTURAL ANALYSIS}

The structural response of a Multi-Degree of Freedom (MDOF) system with $N$ d.o.f. is described by the dynamic equation of equilibrium

$$
\boldsymbol{M} \ddot{\mathbf{Q}}(t, \boldsymbol{u})+\boldsymbol{C} \dot{\boldsymbol{Q}}(t, \boldsymbol{u})+\boldsymbol{F}[\boldsymbol{Q}(t, \boldsymbol{u})]=-\boldsymbol{M} \boldsymbol{\tau} \ddot{X}_{g}(t, \boldsymbol{u})
$$

where $\boldsymbol{Q}(t, \boldsymbol{u})$ is the relative displacement, $\boldsymbol{M}$ and $\boldsymbol{C}$ are mass and damping matrices, $\boldsymbol{F}[\boldsymbol{Q}(t, \boldsymbol{u})]$ is the restoring force vector, $\boldsymbol{\tau}$ is an vector of unit entries, $\ddot{X}_{g}(t, \boldsymbol{u})$ is the stochastic ground acceleration. The force $\boldsymbol{F}[\boldsymbol{Q}(t, \boldsymbol{u})]$ describes the non-linear response of the structural system during strong earthquakes through appropriate hysteresis models.

Let $X(t, \boldsymbol{u})$ be the stochastic response defining the EDP. A typical value used in PBEE is the interstory drift, so that $X(t, \boldsymbol{u})=Q_{1}(t, \boldsymbol{u})$ or $X(t, \boldsymbol{u})=Q_{k}(t, \boldsymbol{u})-Q_{k-1}(t, \boldsymbol{u})$, $k>1$, where $Q_{k}(t, \boldsymbol{u})$ is the $k$-th stochastic relative displacement. The tail probability is defined as the probability that the stochastic response $X(t, \boldsymbol{u})$ of the system is greater than a chosen treshold $x$ at time instant $t$, that is $P_{f}=\operatorname{Prob}[X(t, \boldsymbol{u}) \geq x]$. To apply the structural reliability theory [22, 23], define the limit state function $g(t, x, \boldsymbol{u})=x-X(t, x, \boldsymbol{u})$, so that $\operatorname{Prob}[g(t, x, \boldsymbol{u}) \leq 0]$ is equal to the tail probability [12]. 
The tail probability can be determined through a Monte Carlo Simulation (MCS), however it is consuming, especially for evaluating the very small tai; probabilities. An optimal tradeoff between accuracy and efficiency is represented from the First Order Reliability Method (FORM). FORM requires the evaluation of the design point, usually given as a solution of a constrained optimization problem, $\boldsymbol{u}^{*}(t, x)=\arg \min \{\|\boldsymbol{u}\|: g(t, x, \boldsymbol{u})=0\}$. The corresponding reliability index reads as $\beta(t, x)=\left\|\boldsymbol{u}^{*}(t, x)\right\|$. The FORM solution $P_{f, F O R M}(t, x)=\Phi[-\beta(t, x)]$ gives the first-order approximation of the tail probability, which in most cases of practical interest is very satisfactory. The application of FORM for several values of the threshold $x$ gives the conditional POE of the EDP evaluated at the time instant $t$, i.e. $P\left[x \mid a_{g}\right]=\operatorname{Prob}\left[X(t, \boldsymbol{u}) \geq x \mid A_{g}=a_{g}\right]$.

For design purposes, the main quantity of interest is the first passage probability of the EDP. It has been recognized [11] that a correspondence one-to-one exists between the design point $\boldsymbol{u}^{*}(t, x)$ and the slope $\boldsymbol{a}(t, x)$ of the hyperplane detected by FORM, namely $\boldsymbol{a}(t, x)=x \boldsymbol{u}^{*}(t, x) /\left\|\boldsymbol{u}^{*}(t, x)\right\|^{2}$. Consequently, the knowledge of the design point allows to determine Tail Equivalent Linear System (TELS), which is the equivalent linear system having for each threshold $x$ the same design point of the original nonlinear system. The response of the TELS is $X_{\text {TELS }}(t, x, \boldsymbol{u})=\boldsymbol{a}(t, x) \cdot \boldsymbol{u}$, which has zero-mean and variance $\operatorname{Var}\left[X_{\text {TELS }}(t, x)\right]=\|\boldsymbol{a}(t, x)\|^{2}$. From the knowledge of $\boldsymbol{a}(t, x)$, at stationarity the TELS can be fully characterized by its PSD given as [24]

$$
G_{X}\left(x, \omega_{i}\right)=\frac{\left\{a_{i}^{c}(t, x)\right\}^{2}+\left\{a_{i}^{s}(t, x)\right\}^{2}}{\Delta \omega}
$$

where $a_{i}^{c}(t, x)$ and $a_{i}^{s}(t, x)$ are the components of $\boldsymbol{a}(t, x)$ corresponding respectively to the normal standard random variables $u_{i}^{c}$ and $u_{i}^{s}$ of Eq.(5). From Eq.(7) the spectral moments of the TELS can be determined

$$
\lambda_{m}(x)=\int_{0}^{\infty} \omega^{m} G_{X}(x, \omega) d \omega \cong \sum_{i} \omega_{i}^{m} G_{X}\left(x, \omega_{i}\right) \Delta \omega, \quad m=0,1,2
$$

which allow to evaluate the first-passage probability $P_{f, \max }(t, x)=\operatorname{Prob}\left[X_{\max }(t, \boldsymbol{u}) \leq x\right]$, where $X_{\max }(t, \boldsymbol{u})=\max _{0 \leq \tau \leq t}|X(\tau, \boldsymbol{u})|, 0 \leq \tau \leq t$. For Gaussian stationary processes, several formulations are available. In TELM the known solution of Vanmarcke [25] is adopted:

$$
P_{f, \max }(t, x) \cong\left\{1-\exp \left[-\frac{r^{2}(x)}{2}\right]\right\} \times \exp \left\{-\frac{t}{\pi} \cdot \sqrt{\frac{\lambda_{2}(x)}{\lambda_{0}(x)}} \cdot \frac{1-\exp \left[-\sqrt{\pi / 2} \delta^{1.2}(x) r(x)\right]}{\exp \left[r^{2}(x) / 2\right]-1}\right\}
$$

where

$$
r(x)=\frac{x}{\sqrt{\lambda_{0}(x)}}, \quad \delta(x)=\sqrt{1-\frac{\lambda_{1}^{2}(x)}{\lambda_{0}(x) \lambda_{2}(x)}}
$$


Extensive numerical experimentation has shown that in many cases of practical interest TELM gives a good approximation of the first-passage probability of the EDP, while the required computational effort is only the evaluation of the design point. The application of Eqs.(7)-(10) for several values of the threshold $x$ gives the required conditional POE of the seismic demand, $P\left[x \mid a_{g}\right]=\operatorname{Prob}\left[X_{\text {max }}(t, \boldsymbol{u}) \geq x \mid A_{g}=a_{g}\right]$, coinciding with the fragility curve corresponding to the considered limit state. Of course, in the framework of the PBEE the POE $P[x]=\operatorname{Prob}\left[X_{\max }(t, \boldsymbol{u}) \geq x\right]$ of the EDP reads as

$$
\begin{aligned}
P[x] & =\sum_{L S} P\left[x \mid a_{g, L S}\right] p\left(a_{g, L S}\right)= \\
& =\operatorname{Prob}\left[X_{\max } \geq x \mid a_{g, D L S}\right] p\left(a_{g, D L S}\right)+\operatorname{Prob}\left[X_{\max } \geq x \mid a_{g, U L S}\right] p\left(a_{g, U L S}\right)
\end{aligned}
$$

\section{NUMERICAL APPLICATION}

Consider a Bouc-Wen [26] hysteretic oscillator defined by the nonlinear differential equations

$$
\begin{aligned}
& m \ddot{X}+c \dot{X}+k[\alpha X+(1-\alpha) Z]=-m \ddot{X}_{g}(t) \\
& \dot{Z}=-\delta|\dot{X}||Z|^{r-1} Z-\gamma|Z|^{r} \dot{X}+A \dot{X}
\end{aligned}
$$

where $m, c$ and $k$ are the mass, damping and stiffness coefficients, $\alpha$ controls the degree of hysteresis, and $r, A, \delta$ and $\gamma$ are parameters defining the shape of the hysteresis loop. The base acceleration $\ddot{X}_{g}(t)$ has been modeled using the representation (5), where the PSD is compatible with the elastic response spectrum of EC8 defined in Eq.(1), by assuming the soil category "B", $\zeta_{0}=5 \%$ as suggested by the codes and a PGA $a_{g}=0.20 \mathrm{~g}$, corresponding to the Ultimate Limit State of a zone of high seismicity. The response-spectrum compatible PSD $G(\omega)$ has been determined through Eqs.(1)-(4), assuming a time observing window $t=30 \mathrm{sec}$, when the system achieves the stationarity. The parameters of the oscillator are $m=30,000 \mathrm{~kg}$, natural frequency is $\omega_{0}=8.37 \mathrm{rad} / \mathrm{sec}$ while the damping ratio is $\zeta_{0}=0.05$. The parameters of the Bouc-Wen model are $\alpha=0.10, n=1, A=1, \delta=\gamma=50$. The frequency step in (5) is chosen as $\Delta \omega=(2 \pi) / t=0.21 \mathrm{rad} / \mathrm{sec}$. The power spectrum density has been discretized from $\omega_{1}=0 \mathrm{rad} / \mathrm{sec}$ to $\omega_{n}=20 \mathrm{rad} / \mathrm{sec}$, giving rise to $n=96$ harmonic components and $2 n=192$ normal standard random variables. As EDP it has been chosen the insterstory drift, in this case coinciding with the displacement $X(t)$ of the SDOF system. The tail probability of $X(t)$ is calculated for 10 normalized threshold values $x / \sigma$ ranging from 0.5 to 5 with an increment of $0.5, \sigma$ being the standard deviation of the response of the system.

The main challenge of FORM is represented from the evaluation of the design point. Here it has been determined using the gradient-free strategy presented in [14], which requires 100 analyses per threshold. The adoption of the theory of the random vibration to the resulting TELS $\boldsymbol{a}(t, x)$ allows to determine the first passage probability for the given ULS. In Figure 1 the tail probability given by TELM (continuous line) through Eqs.(7)-(10) is compared with MCS with 100,000 samples (circle markers). The figures show the good accuracy also in the range of the small probabilities. From the knowledge of $P_{f, \max }(t, x)$ it follows the determina- 
tion of the seismic fragility curve $P\left[x \mid a_{g}\right]=\operatorname{Prob}\left[X_{\max }(t, \boldsymbol{u}) \geq x \mid A_{g}=a_{g}\right]$, being $a_{g}=0.20 \mathrm{~g}=1.96 \mathrm{~m} / \mathrm{sec}^{2}$. The POE of the EDP in this case is simply given as $P(x)=P\left[x \mid a_{g}\right] p\left(a_{g}\right)=0.10 \cdot \operatorname{Prob}\left[X_{\max }(t, \boldsymbol{u}) \geq x\right]$. If needed, the POE of the EDP can take into account several limit states, through the adoption of Eq.(11).

(a)

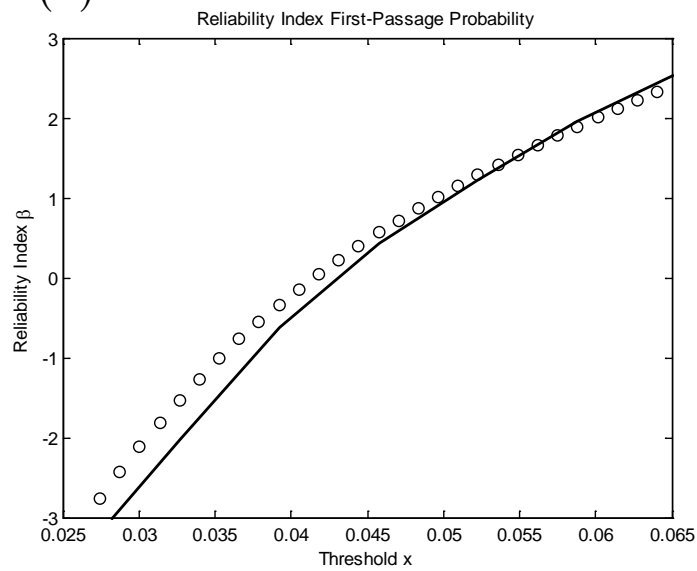

(b)

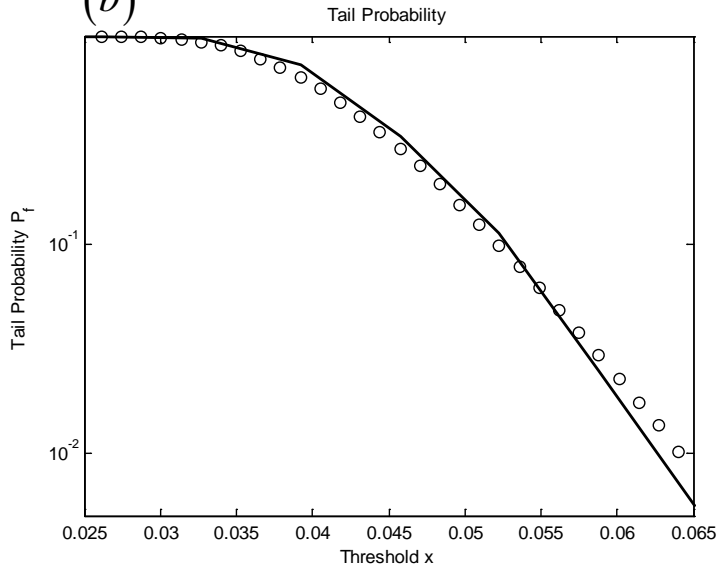

Figure 1: First passage probability of the hysteretic system espressed in terms of (a) reliability index, (b) Tail Probability

\section{CONCLUSIONS}

Performance Based Earthquake Engineering (PBEE) is a probabilistic framework which allows to develop earthquake risk assessment of facilities considering seismic hazard, structural response, damage and losses. In this paper the seismic hazard has been modelled through a stationary Gaussian stochastic process whose PSD is compatible with the elastic response spectrum given by the codes. The seismic fragility curves have been determined through the tools of the Stochastic Dynamic Analysis. It has been adopted the well known First Order Reliability Method (FORM) which gives rise to the Tail Equivalent Linearization Method (TELM). TELM allows to determine the seismic demand of the response with a reduced computational effort. In the framework of PBEE the method can be easily extended for the evaluation of the damage measure and corresponding performance metrics that are of immediate use to both engineers and stakeholders. A more realistic analysis should take into account the nonstationarity of the stochastic ground motion model, and this issue will be analyzed in forthcoming papers. Moreover, in this paper the procedure has been presented to determine the seismic fragility curves of a simple Bouc-Wen Single Degree of Freedom (SDOF) system. Further research will be devoted to check the accuracy and the effectiveness of TELM to more realistic MDOF systems.

\section{ACKNOWLEDGMENTS}

This research is funded by the National Research Foundation Singapore under its Campus for Research Excellence and Technological Enterprise (CREATE) programme

\section{REFERENCES}


[1] B.R. Ellingwood, Earthquake risk assessment of building structures, Reliability Engineering and System Safety, 74, 251-262, 2001

[2] C.A. Cornell \& H. Krawinkler, Progress and challenges in seismic performance assessment, PEER Center News, Spring 2000.

[3] C. Goulet, C.B. Haselton, J. Mitrani-Reiser, J.L. Beck, G.G. Deierlein, K.A. Porter, J.P. Stewart, Evaluation of the seismic performance of a code-conforming reinforcedconcrete frame building - from seismic hazard to collapse safety and economic losses, Earthquake Engineering and Structural Dynamics, 36, 1973-1997, 2007.

[4] T.Y. Yang, J. Moehle, B. Stojadinovic \& A. Der Kiureghian, Seismic performance evaluation of facilities: methodology and implementation, Journal of Structural Engineering ASCE, 135, 1146-1154, 2009.

[5] M.S. Günay \& K.M. Mosalam, PEER Performance-based earthquake engineering methodology, revisited, Journal of Earthquake Engineering, 17(6), 829-858, 2013

[6] D. Vamvatsikos \& C.A. Cornell, Incremental Dynamic Analysis, Earthquake Engineering and Structural Dynamics, 31, 491-514, 2002.

[7] A. Der Kiureghian \& K. Fujimura, Nonlinear stochastic dynamic analysis for performance-based earthquake engineering, Earthquake Engineering and Structural Dynamics, 38, 719-738, 2009

[8] P. Cacciola, P. Colajanni \& G. Muscolino, Combination of modal responses consistent with seismic input representation, Journal of Structural Engineering (ASCE), 130(1), 47-55, 2004.

[9] M. Di Paola \& M. Navarra, Stochastic seismic analysis of MDOF structures with nonlinear viscous dampers, Structural Control and Health Monitoring, 16, 303-318, 2008.

[10] S.K. Au \& J.L. Beck, Subset simulation and its application to seismic risk based based on dynamic analysis, Journal of Engineering Mechanics (ASCE), 129, 901-917, 2003.

[11] K. Fujimura \& A. Der Kiureghian, Tail-equivalent linearization method for nonlinear random vibration, Probabilistic Engineering Mechanics, 22, 63-76, 2007.

[12] A. Der Kiureghian, The geometry of random vibration and solutions by FORM and SORM, Probabilistic Engineering Mechanics, 15, 81-90, 2000.

[13] S.O. Rice. Mathematical analysis of random noise, Selected papers on Noise and Stochastic Processes, Dover Publications Inc. New York, 1954

[14] U. Alibrandi, A Response Surface Method for stochastic dynamic analysis, Reliability Engineering and System Safety, 26, 44-53, 2014.

[15] T. Haukaas \& A. Der Kiureghian. Finite Element Reliability and Sensitivity Methods for Performance-Based Earthquake Engineering, Peer Report 2003/04, Pacific Earthquake Engineering Research Center, University of California, Berkeley, CA.

[16] T. Haukaas \& A. Der Kiureghian, Strategies for finding the design point in non-linear finite element reliability analysis, Probabilistic Engineering Mechanics, 21, 133-147, 2006.

[17] F. Mckenna, G.L. Fenves, M.H. Scott, Open system for earthquake engineering simulation. Pacific Earthquake Engineering Research Center, University of California, Berkeley, CA, 2003, http://opensees.berkeley.edu 
[18] U. Alibrandi \& A. Der Kiureghian, A Gradient-Free Method for Determining the Design Point in Nonlinear Stochastic Dynamic Analysis, Probabilistic Engineering Mechanics, 28, 2-10, 2012.

[19] P. Cacciola, L. D’Amico \& I. Zentner, New insights in the analysis of the structural response to response-spectrum-compatible accelerograms, Engineering Structures, 78, 316, 2014

[20] M. Shinozuka, Digital simulation of random processes, Journal of Sound and Vibration, 25, 111-128, 1972

[21] M. Shinozuka \& G. Deodatis, Simulation of stochastic process by spectral representation, American Society of Mechanical Engineers, 25, 191-204, 1991.

[22] O. Ditlevsen \& H.O. Madsen. Structural Reliability Methods, Wiley. 1996.

[23] R.E. Melchers, Structural Reliability, analysis and prediction. New York: Wiley \& Sons, 1999.

[24] L. Garrè \& A. Der Kiureghian, Tail Equivalent Linearization Method in frequency domain and application to marine structures, Marine Structures, 23, 322-338, 2010.

[25] E. Vanmarcke, On the distribution of the first-passage time for normal stationary random processes, Journal of Applied Mechanics, 42, 215-220, 1975.

[26] JK Wen, Approximate method for non-linear random vibration, J Engng Div, ASCE, 101, 389-401, 1975 